\title{
Doppler Tomography in 2D and 3D of the X-ray Binary Cyg X-1 for June 2007
}

\author{
O. I. Sharova ${ }^{1}$, M. I. Agafonov ${ }^{1}$, E. A. Karitskaya ${ }^{2}$, N. G. Bochkarev ${ }^{3}$, \\ S. V. Zharikov ${ }^{4}$, G. Z. Butenko ${ }^{5}$, and A. V. Bondar ${ }^{5}$ \\ ${ }^{1}$ Radiophysical Research Institute, B.Pecherskaya str, 25/12a, Nizhny Novgorod 603950 Russia \\ ${ }^{2}$ Institute of Astronomy RAS, Moscow 119017 Russia \\ ${ }^{3}$ Sternberg Astronomical Institute 13 Universitetskij pr., Moscow 119991 Russia \\ ${ }^{4}$ Mexican National Astronomical Observatory, Institute of Astronomy, UNAM, Mexico \\ ${ }^{5}$ International Center for Astronomical, Medical and Ecological Research, Terscol, \\ Kabarda-Balkarian Republic, 361605 Russia
}

\begin{abstract}
The 2D and 3D Doppler tomograms of X-ray binary system Cyg X-1 (V1357 Cyg) were reconstructed from spectral data for the line HeII $4686 \AA$ obtained with 2-m telescope of the Peak Terskol Observatory (Russia) and 2.1-m telescope of the Mexican National Observatory in June, 2007. Information about gas motions outside the orbital plane, using all of the three velocity components $V_{x}, V_{y}, V_{z}$, was obtained for the first time. The tomographic reconstruction was carried out for the system inclination angle of $45^{\circ}$. The equal resolution $(50 \times 50 \times 50 \mathrm{~km} / \mathrm{s})$ is realized in this case, in the orbital plane $\left(V_{x}, V_{y}\right)$ and also in the perpendicular direction $V_{z}$. The checkout tomograms were realized also for the inclination angle of $40^{\circ}$ because of the angle uncertainty. Two versions of the result showed no qualitative discrepancy. Details of the structures revealed by the 3D Doppler tomogram were analyzed.
\end{abstract}

Keywords. Accretion, accretion disks - binaries: x-ray - binaries: imaging - stars: individual (Cyg X-1) - techniques: image processing

The realization of the 3D Doppler tomography became possible due to the development of the Radioastronomical Approach for reconstruction in the case of few projections (Agafonov \& Sharova 2005a; Agafonov \& Sharova 2005b). A direct comparison was made between the observed spectra and those computed from the constructed 3D Doppler tomograms. Chi-square statistics show the good quality of the reconstruction. The number of Mexican observation profiles (83) was larger than the 51 spectra from Terskol, so we used mainly the results of the 3D Mexican Doppler tomogram, and the Terskol results were used only as a test. However, there was good similarity in the discovered features.

Two-Dimensional standard Doppler tomograms for 2007 June are similar to the earlier reconstructed tomograms for 1997, 2003 and 2004 (Karitskaya et al., 2005; Karitskaya et al., 2007). They show: A) the emission component of the HeII $4686 \AA$ line is generated mainly in the outer parts of the accretion structure closest to the donor star (O-supergiant) and from the optically thick accretion disk; B) the absorption component is the feature of the O-supergiant atmosphere.

Three-Dimensional tomograms are reconstructed in $3 \mathrm{D}$ velocity space $\left(V_{x}, V_{y}, V_{z}\right)$. Their structure shows that the formation of He II $4686 \AA$ line profiles is also connected both with the area of accretion structure (see Fig. 1 and 2) and with the donor-star (supergiant). However, some additional features have been discovered.

A. The first predominant feature is the emission component located around the central slice. This area consists of individual feature components with different $V_{z}$. But all these $V_{z}$ values lie within the limits of -200 to $+160 \mathrm{~km} / \mathrm{s}$. Here we can see a combination of three main emission feature components: 1) the emission of the outer part of the accretion 

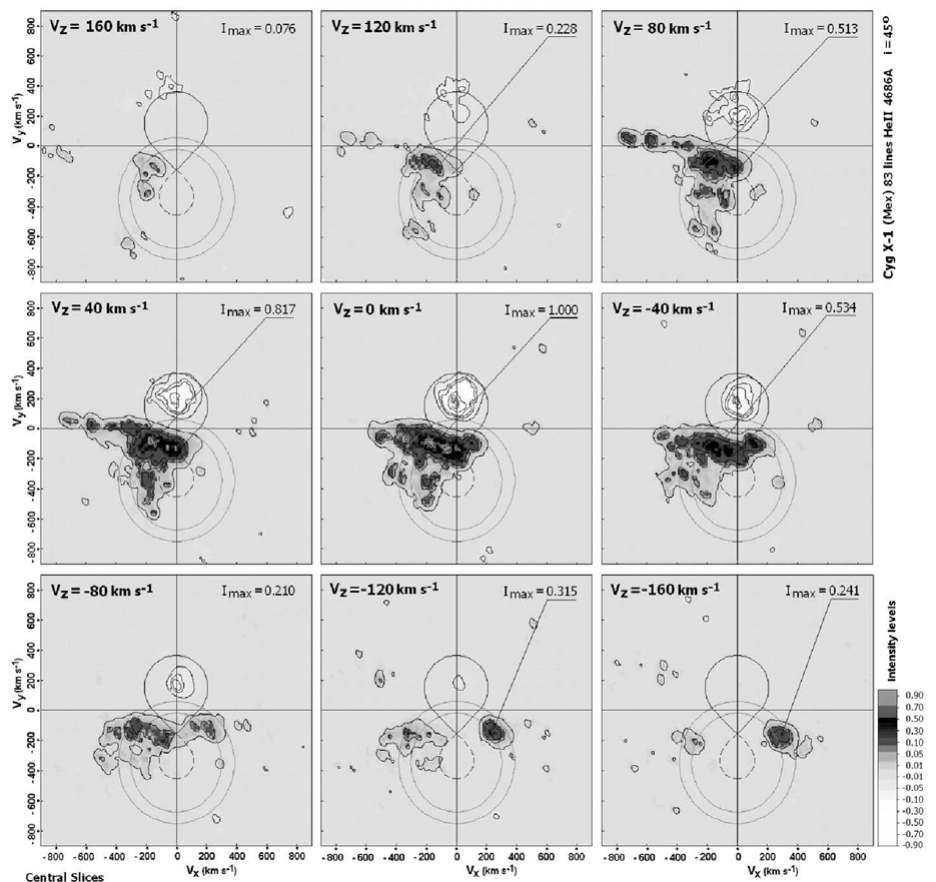

Figure 1. Nine central slices of the Cyg X-1 3D Doppler tomogram in the $\left(V_{x}, V_{y}\right)$ plane for different $V_{z}$. The outlines of the Roche lobe of the donor star and the pattern of the outer parts of the accretion disk are plotted on the slices. We used the mass ratio of $q=M_{x} / M_{O}=1 / 3$ and two versions of the disk size.
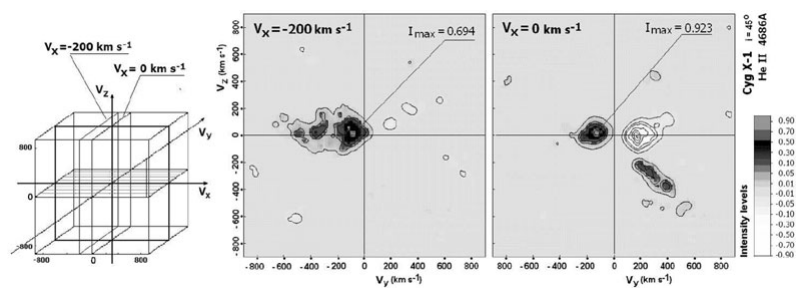

Figure 2. Cross-sections of of the Cyg X-1 3D Doppler tomogram in the $\left(V_{y}, V_{z}\right)$ plane for two different $V_{x}$. The cube shows the geometry of the arrangement of the slices in $3 \mathrm{D}$ velocity space.

structure near the donor-star; 2) the elongated feature of the stream emerges from the L1 point; 3) the emission produced by the stream - accretion structure interaction.

B. The other predominant feature is visible in absorption and is associated with the supergiant. It is a compact structure. The maximum of absorption corresponds to the central slice $\left(V_{x}, V_{y}\right)$ of the tomogram with $V_{z}=0 \mathrm{~km} / \mathrm{s}$. There is also an interesting emission feature identified with a structure related with the supergiant (in the co-rotation coordinate system). It has a $V_{z} \sim-200$ to $-400 \mathrm{~km} / \mathrm{s}$ and an intensity $I \sim 20-30 \%$ of the maximum 3D tomogram intensity (see Fig. 2). That is probably a stream from the donor-star visible in emission flowing almost perpendicularly to the orbital plane.

This work was partially supported by RFBR (grants 09-02-01136 and 09-02-00993).

\section{References}

Agafonov, M. I. \& Sharova, O. I. 2005a, Radiophys. Quant. Electron., 48, 5, 329

Agafonov M. I. \& Sharova, O. I. 2005b, AN, 326, 143

Karitskaya, E. A., Agafonov, M. I., Bochkarev, N. G. et al., 2005, A\&\&A Trans., 24, 383

Karitskaya, E. A., Agafonov, M. I., Bochkarev, N. G. et al., 2007, A\&A Trans., 26, 159 